Laís da Mata Almeida

Investigação do Uso de Hidroxiácidos para Adesão em Esmalte e em Dentina

Brasília

2018 

Laís da Mata Almeida

\section{Investigação do Uso de Hidroxiácidos para Adesão em Esmalte e em Dentina}

Trabalho de Conclusão de Curso apresentado ao Departamento de Odontologia da Faculdade de Ciências da Saúde da Universidade de Brasília, como requisito parcial para a conclusão do curso de Graduação em Odontologia.

Orientador: Prof. Dr. Leandro Augusto Hilgert

Co-orientadora: Prof. Dra. Cristina de Mattos Pimenta Vidal

Brasília 

A Deus, pelo dom da vida.

À minha família, pela confiança 



\section{AGRADECIMENTOS}

A Deus, por ter me ensinado a lutar, a não temer, e a nunca desistir dos meus sonhos.

Aos meus pais, Celimar e Leonardo, pelo amor, pelo suporte dado desde a minha infância e por terem acreditado em mim, mais do que eu mesma fosse capaz.

À minha irmã, Júllia, por ser a minha inspiração diária.

À Bianca e à Raíssa, minhas grandes amigas, por compartilharem momentos únicos e por estarem sempre ao meu lado.

Ao meu orientador, Leandro Augusto Hilgert, pelo suporte, por ter acreditado em mim e por ter me oferecido grandes oportunidades desde o início do curso.

À minha dupla de pesquisa, Daniela, pelo auxílio neste trabalho de conclusão de curso.

À grandiosa dentista, Akele Diniz por ter me apresentado a verdadeira odontologia, pela paciência, pelo cuidado, pela oportunidade e pelos ensinamentos dados.

Ao meu companheiro e namorado, Tiago, que foi o maior presente que a faculdade me proporcionou, me permitindo finalizar o curso de uma maneira memorável. 



\section{EPÍGRAFE}

"Descobri como é bom chegar quando se tem paciência. E, para se chegar onde quer que seja, aprendi que não é preciso dominar a força, mas a razão. É preciso, antes de tudo, querer." 

RESUMO

ALMEIDA, Laís da Mata. Investigação do Uso de Hidroxiácidos para Adesão em Esmalte e em Dentina, 2018. Trabalho de Conclusão de Curso (Graduação em Odontologia) Departamento de Odontologia da Faculdade de Ciências da Saúde da Universidade de Brasília.

Este estudo investigou o uso de diferentes hidroxiácidos para condicionamento do esmalte e da dentina na resistência de união $(R U)$ da interface dente/resina composta. 40 terceiros molares humanos tiveram dentina exposta e planificada. 40 incisivos bovinos tiveram o esmalte planificado. Os dentes foram aleatorizados em 5 grupos e condicionados 30 s em esmalte e 15 s em dentina com os seguintes ácidos a 37\%: fosfórico (FOS, controle), glicólico (GLI), tartárico (TAR), gluconolactona (GLA) ou glucônico (GLU). Os molares (dentina) foram restaurados com o sistema adesivo Single Bond 2 (3M) e resina composta Z250(3M) e seccionados na forma de palitos para o teste de microtração ( $\mu$ TBS). Os incisivos (esmalte) foram hibridizados com o mesmo adesivo e sobre a superfície construídos cilindros de resina $(\varnothing=0,8 \mathrm{~mm})$ para teste de microcisalhamento ( $\mu S B S$ ). Os testes de $\mu$ TBS e $\mu$ SBS foram realizados e os padrões de fratura analisados sob microscopia. Os dados foram submetidos à análise de variância de um fator - ANOVA e "post hoc" de Tukey $(\alpha=0,05)$. Os resultados indicaram que em esmalte, os grupos FOS, GLI e TAR apresentaram os valores mais altos de $\mathrm{RU}$. Os grupos FOS e GLI foram significativamente superiores aos grupos GLU e GLA. Em dentina, os grupos GLI, FOS, TAR e GLU apresentaram os valores de RU mais elevados. Os grupos GLI e FOS apresentaram valores significativamente superiores ao grupo GLA. Assim, conclui-se que o condicionamento de 
esmalte e dentina pode ser realizado por hidroxiácidos alternativos ao ácido fosfórico. Entre os hidroxiácidos testados, destacam-se os valores de RU promovida pelos ácidos glicólico e tartárico, semelhante a do ácido fosfórico. 


\section{ABSTRACT}

ALMEIDA, Laís da Mata. Investigation on the Use of Hydroxy Acids for Adhesion to Enamel and Dentin, 2018. Undergraduate Course Final Monograph (Undergradute Course in Dentistry) Department of Dentistry, School of Health Sciences, University of Brasilia.

This study tested different hydroxy acids used for enamel and dentin conditioning in the bond strength of the tooth / composite resin interface. 40 third human molars had dentin exposed and flattened. 40 bovine incisors had enamel flattened. Teeth were randomized in 5 groups and conditioned (30s, enamel; $15 \mathrm{~s}$ dentin) with the following acids: phosphoric (FOS, control), glycolic (GLI), tartaric (TAR), gluconolactone (GLA) or gluconic (GLU). The human molars (dentin) were restored with the adhesive system Single Bond 2 (3M) and composite resin Z250 (3M) and cut into sticks for the microtensile bond strength test ( $\mu$ TBS). The bovine incisors (enamel) were hybridized with the same adhesive. On the surface composite resin cylinders $(\varnothing=$ $0.8 \mathrm{~mm}$ ) were constructed for the microshear bond strength test ( $\mu \mathrm{SBS})$. The $\mu$ TBS and $\mu$ SBS tests were performed and the fracture patterns analyzed under microscopy. Data were submitted to Analysis of Variance - ANOVA and Tukey "post hoc" $(\alpha=0.05)$. The results showed that on enamel, FOS, GLI and TAR groups presented the highest values of $\mu$ SBS. The FOS and GLI groups were significantly superior to the GLU and GLA groups.On dentin, the GLI, FOS, TAR and GLU groups showed the highest $\mu$ TBS values. The GLI and FOS groups presented values significantly higher than the GLA group. Thus, it can be concluded that the enamel and dentin conditioning can be performed by hydroxy acids that are alternative to phosphoric acid. Among the hydroxy acids tested, the bond strenght values 
of glycolic and tartaric acids, similar to that of phosphoric acid, stands out. 
SUMÁRIO

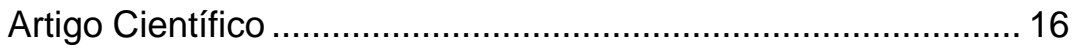

Folha de Título ..................................................................... 18

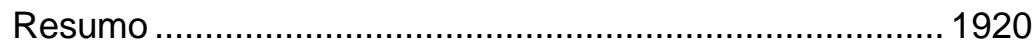

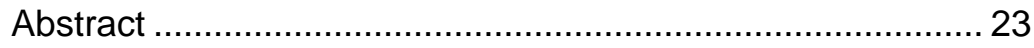

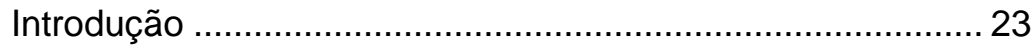

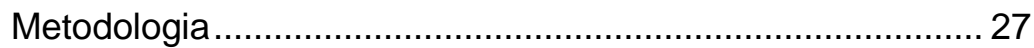

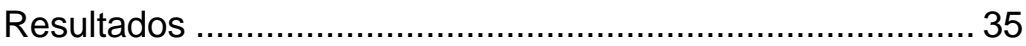

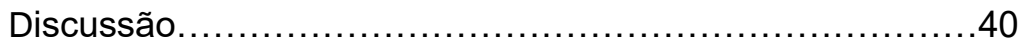

Considerações finais ................ Erro! Indicador não definido.3

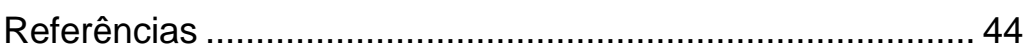

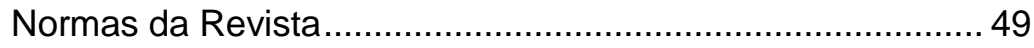





\section{Artigo Científico}

Este trabalho de Conclusão de Curso é baseado no artigo científico:

ALMEIDA, Laís da Mata; VIDAL, Cristina de Mattos Pimenta; HILGERT, Leandro Augusto. Investigação do Uso de Hidroxiácidos para Adesão em Esmalte e em Dentina.

Apresentado sob as normas de publicação da Revista Journal of Dentistry. 


\section{FoLHA DE TÍTULO}

Investigação do Uso de Hidroxiácidos para Adesão em Esmalte e em Dentina.

Investigation on the use of Hydroxy Acids for Adhesion to Enamel and Dentin

Laís da Mata Almeida ${ }^{1}$

Leandro Augusto Hilgert ${ }^{2}$

Cristina de Mattos Pimenta Vidal ${ }^{3}$

1 Aluna de Graduação em Odontologia da Universidade de Brasília.

2 Professor Adjunto de Dentística da Universidade de Brasília.

${ }^{3}$ Professora Adjunta de Dentística da University of lowa.

Correspondência: Prof. Dr. Leandro Augusto Hilgert Campus Universitário Darcy Ribeiro - UnB - Faculdade de Ciências da Saúde - Departamento de Odontologia - 70910-900 Asa Norte - Brasília - DF

E-mail: leandrohilgert@gmail.com / Telefone: (61) 31071849 


\section{Resumo}

Investigação do Uso de Hidroxiácidos para Adesão em Esmalte e em Dentina.

\section{Resumo}

Este estudo investigou o uso de diferentes hidroxiácidos para condicionamento do esmalte e da dentina na resistência de união $(R U)$ da interface dente/resina composta. 40 terceiros molares humanos tiveram dentina exposta e planificada. 40 incisivos bovinos tiveram o esmalte planificado. Os dentes foram aleatorizados em 5 grupos e condicionados 30 s em esmalte e 15 s em dentina com os seguintes ácidos a 37\%: fosfórico (FOS, controle), glicólico (GLI), tartárico (TAR), gluconolactona (GLA) ou glucônico (GLU). Os molares (dentina) foram restaurados com o sistema adesivo Single Bond 2 (3M) e resina composta Z250(3M) e seccionados na forma de palitos para o teste de microtração ( $\mu$ TBS). Os incisivos (esmalte) foram hibridizados com o mesmo adesivo e sobre a superfície construídos cilindros de resina $(\varnothing=0,8 \mathrm{~mm})$ para teste de microcisalhamento ( $\mu S B S$ ). Os testes de $\mu$ TBS e $\mu$ SBS foram realizados e os padrões de fratura analisados sob microscopia. Os dados foram submetidos à análise de variância de um fator - ANOVA e "post hoc" de Tukey $(\alpha=0,05)$. Os resultados indicaram que em esmalte, os grupos FOS, GLI e TAR apresentaram os valores mais altos de $\mathrm{RU}$. Os grupos FOS e GLI foram significativamente superiores aos grupos GLU e GLA. Em dentina, os grupos GLI, FOS, TAR e GLU apresentaram os valores de RU mais elevados. Os grupos GLI e FOS apresentaram valores significativamente superiores ao grupo GLA. Assim, conclui-se que o condicionamento de esmalte e dentina pode ser realizado por hidroxiácidos alternativos ao ácido fosfórico. Entre os hidroxiácidos testados, 
destacam-se os valores de RU promovida pelos ácidos glicólico e tartárico, semelhante a do ácido fosfórico.

Palavras - Chaves: Esmalte, Dentina, Condicionamento Ácido, Hidroxiácidos. 


\section{ABStRACT}

Investigation on the Use of Hydroxy Acids for Adhesion to Enamel and dentin

\section{Abstract}

This study tested different hydroxy acids used for enamel and dentin conditioning in the bond strength of the tooth / composite resin interface. 40 third human molars had dentin exposed and flattened. 40 bovine incisors had enamel flattened. Teeth were randomized in 5 groups and conditioned (30s, enamel; 15s dentin) with the following acids: phosphoric (FOS, control), glycolic (GLI), tartaric (TAR), gluconolactone (GLA) or gluconic (GLU). The human molars (dentin) were restored with the adhesive system Single Bond $2(3 \mathrm{M})$ and composite resin Z250 (3M) and cut into sticks for the microtensile bond strength test ( $\mu$ TBS). The bovine incisors (enamel) were hybridized with the same adhesive. On the surface composite resin cylinders $(\varnothing=$ $0.8 \mathrm{~mm}$ ) were constructed for the microshear bond strength test ( $\mu$ SBS). The $\mu$ TBS and $\mu$ SBS tests were performed and the fracture patterns analyzed under microscopy. Data were submitted to Analysis of Variance - ANOVA and Tukey "post hoc" $(\alpha=0.05)$. The results showed that on enamel, FOS, GLI and TAR groups presented the highest values of $\mu$ SBS. The FOS and GLI groups were significantly superior to the GLU and GLA groups. On dentin, the GLI, FOS, TAR and GLU groups showed the highest $\mu$ TBS values. The GLI and FOS groups presented values significantly higher than the GLA group.Thus, it can be concluded that the enamel and dentin conditioning can be performed by hydroxy acids that are alternative to phosphoric acid. Among the hydroxy acids tested, the bond strenght values of glycolic and tartaric acids, similar to that of phosphoric acid, stands out. 
Keywords: Enamel, Dentin, Acid Conditioning, Hidroxy Acids. 


\section{INTRODUÇÃO}

A Odontologia Adesiva é baseada no uso de materiais restauradores que são aplicados nos tecidos dentais, a fim de promover adesão por meio de retenção micromecânica. Essa adesão depende do condicionamento ácido, uma vez que são criadas microporosidades na superfície dentária ${ }^{1,30}$

Com o passar dos anos, houve um aprimoramento dos materiais adesivos para uso odontológico, permitindo importantes avanços na técnica restauradora, viabilizando procedimentos mais conservadores e estéticos².

Apesar dos avanços nas formulações desses materiais nos últimos anos, a obtenção de interfaces adesivas estáveis e com durabilidade ainda é um desafio. Independente da técnica adesiva utilizada, as restaurações continuam a apresentar falhas nos tecidos dentários ${ }^{1}$, principalmente para os sistemas mais simplificados (convencionais de dois passos e autocondicionantes de passo único) ${ }^{3,4,26,35}$, uma vez que esses, após a polimerização, funcionam como membranas permeáveis permitindo a absorção de água, e consequentemente a degradação dos monômeros resinosos de modo mais fácil. ${ }^{5,34,35}$

Mesmo com a evolução desses materiais, pouco se desenvolveu em relação ao protocolo de condicionamento ácido dos tecidos dentais, que é a etapa inicial do mecanismo de adesão ao esmalte e à dentina pela técnica etch and rinse (convencional), e padronizado pela utilização de ácido fosfórico $37 \%$ em diferentes tempos para cada tecido ${ }^{4,33,35}$.

Em esmalte, esse procedimento tem se mostrado bastante efetivo para promover desmineralização superficial do tecido, resultando na criação de microporosidades para retenção micromecânica dos materiais adesivos.

Além disso, o condicionamento ácido com ácido fosfórico promove a remoção do cálcio da hidroxiapatita, que realiza uma 
união química com alguns compostos presentes em adesivos (10-MDP). ${ }^{3}$

Assim, ultimamente, o condicionamento ácido tem sido recomendando apenas para o esmalte tanto para sistemas adesivos autocondicionantes quanto para sistemas adesivos universais.

No entanto, em dentina, a união dos materiais resinosos é dificultada pela composição orgânica dessa região. Nesse tecido, o condicionamento com ácido fosfórico promove remoção total dos cristais de apatita, que representam o conteúdo mineral dessa superfície, dissolvendo minerais nos espaços inter e intramicrofibrilares; além de expor a malha de colágeno (conteúdo orgânico) para subsequente infiltração dos monômeros resinosos $\mathrm{s}^{4,6,7,8}$

A dificuldade em obter união de monômeros resinosos hidrófobos à dentina justificam os problemas de adesão nesse tecido $^{3,9}$. Dessa forma, os efeitos do condicionamento com ácido fosfórico na dentina podem estar relacionados ao insucesso das restaurações adesivas.

O primeiro aspecto a ser considerado é que um dos mecanismos responsáveis pela instabilidade das interfaces adesivas é a degradação do tecido dentinário a longo prazo ${ }^{1,2,9,10,11,12}$, que ocorre nas áreas de dentina parcialmente desmineralizada exposta, em função da incompleta infiltração dos monômeros resinosos ${ }^{12,13}$.

Essa degradação ocorre por uma atividade proteolítica endógena atribuída às metaloproteinases da matriz (MMPs) e as cisteíno-catepsinas (CTs) $)^{11,13,14,15}$.

As metaloproteinases e as cisteíno-catepsinas (CTs) são secretadas por anticorpos e degradam colágeno. Como a dentina possui colágeno, acredita-se que essas enzimas possam ser ativadas pelo $\mathrm{pH}$ durante 0 condicionamento ácido ${ }^{15,16}$ fragilizando ainda mais a dentina. 
Além dos efeitos na atividade de proteases, o uso de ácido fosfórico remove proteoglicanos da matriz dentinária ${ }^{17}$, podendo alterar o controle de água na matriz, e também a atividade dessas moléculas ${ }^{18,19,20}$.

Para completar, o protocolo de condicionamento com ácido fosfórico causa alterações na estrutura do colágeno, promovendo sua desnaturação ou desdobramento da alfa-hélice da molécula ${ }^{21}$, o que pode facilitar sua degradação proteolítica ${ }^{18}$.

Outra condição a ser citada é a completa remoção de minerais promovida pelo ácido fosfórico, impedindo a potencial união química de alguns monômeros resinosos ao cálcio da hidroxiapatita. A desmineralização intensa também remove conteúdo mineral localizado em regiões intrafibrilares, responsável pela resistência mecânica do tecido ${ }^{22,23}$.

$\mathrm{O}$ quadro a seguir indica os efeitos de se ter o ácido fosfórico em dentina e em esmalte.

Quadro 1: Quadro indicador dos efeitos do ácido fosfórico no dente.

\begin{tabular}{|l|l|}
\hline \multirow{3}{*}{ Esmalte } & Ácido Fosfórico \\
\hline & $\begin{array}{l}\text { - Microporosidades são criadas permitindo } \\
\text { uma adesão adequada, uma vez que o } \\
\text { esmalte não é úmido e não tem uma região } \\
\text { orgânica significativa. }\end{array}$ \\
\hline \multirow{5}{*}{ Dentina } & $\begin{array}{l}\text { - Remoção total do conteúdo mineral na } \\
\text { superfície; } \\
\text { - Dissolve minerais nos espaços fibrilares; } \\
\text { - Expõe a malha de colágeno; } \\
\text { - O pH do ácido fosfórico ativa } \\
\text { metaloproteinases da matriz (MMPs) e } \\
\text { cisteíno-catepsinas (CTs) que degradam os } \\
\text { proteoglicanos da dentina; } \\
- \text { Alterações na estrutura do colágeno, } \\
\text { promovendo sua desnaturação ou } \\
\text { desdobramento da alfa-hélice da molécula. }\end{array}$ \\
\hline
\end{tabular}


Foram investigados ácidos orgânicos polifuncionais ou hidróxiácidos, incluindo alfa-hidróxiácidos $(\mathrm{AHAs})^{29}$, betahidróxiácidos (BHAs), poli-hodróxiácidos (PHAs) e ainda os ácidos biônicos (BA).

Os hidroxiácidos são compostos que podem ser encontrados na natureza em plantas, frutas e microorganismos participando de alguns processos metabólicos. São amplamente utilizados na dermatologia desde a década de 70 para tratamento de dermatoses, hiperpigmentação, acne, fotoenvelhecimento e em procedimentos como peeling facial ${ }^{24}$. Alguns desses ácidos possuem potencial antioxidante ${ }^{25}$, aumentam a síntese de colágeno ${ }^{23} \mathrm{e}$ inibem a atividade das $\mathrm{MMPs}^{25}$.

Os hidroxiácidos (ácido glicólico, ácido tartárico, ácido glucônico e gluconolactona) foram utilizados no protocolo restaurador em esmalte e em dentina associados a um sistema adesivo convencional e os resultados foram comparados aos obtidos com o ácido fosfórico, representando o grupo controle.

Desta forma, esta pesquisa propõe a utilização de outros ácidos para condicionamento do esmalte e da dentina, a fim de estabelecer métodos alternativos que sejam mais conservadores, promovam menor modificação dos componentes orgânicos da matriz dentinária e que resultem em adequada adesão aos tecidos dentais. 


\section{Metodologia}

Coleta de Dentes Humanos e de Dentes Bovinos

Através de doações de pacientes atendidos na clínica do Departamento de Odontologia da Universidade de Brasília, com a aprovação pelo Comitê de Ética em Pesquisa da Universidade de Brasília - UnB (Brasília, Distrito Federal, Brasil) (CAAE 69919516.0.0000.0030), foram coletados 48 terceiros molares humanos recém extraídos. Simultaneamente, foram coletados 48 incisivos bovinos obtidos de frigorífico. Ambos os grupos de dentes foram armazenados em freezer à $-20^{\circ} \mathrm{C}$.

\section{Preparo das Amostras}

Após a obtenção dos 96 dentes humanos e bovinos, foi feita uma seleção aleatória das amostras, na qual se escolheu 40 molares humanos e 40 incisivos bovinos $(n=80)$.

Os molares sofreram testes de microtração com condicionamento através de hidróxiacidos em dentina; e os incisivos bovinos, os testes de microcisalhamento com hidroxiácidos em esmalte.

Dos 16 dentes restantes, sendo 8 bovinos e 8 humanos, 2 dentes de cada espécie foram utilizados como meio de treinamento para o início das amostras em um estudo piloto. Os 12 dentes restantes foram colocados como dentes extras para possíveis necessidades e armazenados em geladeira à $5^{\circ} \mathrm{C}$.

O ácido fosfórico (FOS) e os hidróxiácidos (ácido glicólico, GLI; ácido tartárico, TAR; ácido glucônico, GLU; e gluconolactona, GLA) foram manipulados à $37 \%$ e medidos em pHmêtro (Hanna, São Paulo, Brasil). Para reagentes líquidos (glucônico e fosfórico), foram misturados $4.12 \mathrm{ml}$ de cada ácido com $5.88 \mathrm{ml}$ de água destilada (Reymer, Aparecida de Goiânia, Brasil). 
Para os reagentes em pó (Glicólico, Gluconalactona e Tartárico), misturou-se $3.7 \mathrm{~g}$ de pó do respectivo ácido com água destilada (Reymer, Aparecida de Goiânia, Brasil) até obter $10 \mathrm{ml}$ de solução.

Os ácidos foram armazenados em potes de vidro e colocados em geladeira à $5^{\circ} \mathrm{C}$, para serem utilizados conforme a necessidade.

\section{Preparo dos Dentes Humanos para Microtração}

Cada molar humano teve sua região oclusal de esmalte seccionada através de uma cortadeira de precisão (Micromet, Rockvill, Estados Unidos da América) com disco diamantado (Erios, São Paulo, Brasil) sob refrigeração à água.

Em seguida, as superfícies dos molares humanos foram desgastadas em máquina Politriz Metalográfica (Teclago, São Paulo, Brasil) com lixa de granulação número 600 (3M ESPE, Minessota, Estados Unidos da América) sob refrigeração com água por 60 segundos contados em cronômetro, para reproduzir de forma padronizada a lama dentinária criada durante o preparo dentário.

Após o desgaste, os dentes foram divididos de acordo com o tipo de ácido e técnica de aplicação a ser utilizada $(n=8)$, sendo assim, 8 dentes para cada hidroxiácido.

O condicionamento ácido foi realizado de forma passiva (mantendo-se o mesmo volume de solução na superfície) através de pipeta e de aplicadores descartáveis com 15 segundos em dentina. Em seguida, foi feita a lavagem com água corrente por 10 segundos. $O$ excesso de umidade foi controlado através de secagem com algodão, e a dentina foi mantida úmida.

Utilizou-se o sistema adesivo Adper Single Bond 2 (3M ESPE, Minnesota, Estados Unidos da América) de forma ativa (esfregou-se 0 adesivo na superfície com micro-pincel descartável) por 15 segundos, seguido de jato de ar de pressão 
contínua para volatilização dos solventes por 15 segundos. Houve uma nova aplicação de adesivo do mesmo modo do anterior. Em seguida, foi feita a fotopolimerização com Bluephase (Ivoclar Vivadent, Barueri, Brasil) do adesivo por 20 segundos com irradiância de $1.200 \mathrm{~mW} / \mathrm{cm}^{2}$.

Após a aplicação do adesivo, cada dente recebeu um incremento de $1 \mathrm{~mm}$ de espessura com resina composta microhíbrida (Filtek Z250, 3M ESPE, Minessota, Estados Unidos da América) que foi fotopolimerizado por 40 segundos.

Seguido dessa primeira camada de $1 \mathrm{~mm}$, foram colocados 3 outros incrementos, sendo cada um com $1 \mathrm{~mm}$ de espessura da resina microhíbrida Charisma (Classic Esmalte A3 4G - Heraeus Kulzer, São Paulo, Brasil), para que obtivéssemos uma restauração com no mínimo $4 \mathrm{~mm}$ de altura.

Para que pudesse ser mensurado o tamanho de cada incremento, foi utilizado um paquímetro. Além disso, as restaurações foram realizadas apenas em região de dentina exposta.

Ao final do procedimento restaurador, os dentes foram armazenados em água destilada (Reymer, Aparecida de Goiânia, Brasil) por 48 horas à temperatura ambiente.

Passado esse período, as amostras de dentes humanos tiveram suas coroas seccionadas na mesma cortadeira de precisão com um disco diamantado sob refrigeração com água para se obter espécimes na forma de palitos com área média de $0.8 \mathrm{~mm}^{2}$.

Conforme os palitos eram produzidos, armazenava-se em água destilada (Reymer, Aparecida de Goiânia, Brasil) para que não houvesse ressecamento das amostras.

Para o ensaio mecânico, os espécimes foram individualmente fixados em um par de dispositivos (Jig Geraldeli 2 - Odeme - Oa52, Flórida, Estados Unidos da América) à uma distância de $0,25 \mathrm{~mm}$ com cola a base de cianocrilato (Super 
Glue Gel, Loctite, Henkel North America, Ohio, Estados Unidos da América).

As amostras provenientes de cada dente restaurado foram utilizadas para testar a resistências de união à microtração ( $\mu$ TBS) imediata. Utilizando um equipamento de testes de microtração. Os testes foram realizados em uma máquina de ensaios universal Shimadzu EX-L (Shimadzu Corp, Quioto, Japão) a uma velocidade de $0.5 \mathrm{~mm} / \mathrm{min}$. As médias e desvios padrão da $\mu$ TBS de cada grupo foram calculados e expressos em MPa.

Os testes de microtração foram realizados com uma média de 5 palitos por dente de forma aleatória. Algumas amostras excederam o número médio palitos e, então, foram armazenados em timol para testes futuros.

Para a produção de 1 litro de timol, foram manipulados $900 \mathrm{~mL}$ de água destilada (Reymer, Aparecida de Goiânia, Brasil), $100 \mathrm{~mL}$ de álcool absoluto 99,5\% (Rioquímica, São José do Rio Preto, Brasil), $9 \mathrm{~g}$ de cloreto de sódio em pó (Synth, SPLabor, Presidente Prudente, Brasil) e $4 \mathrm{~g}$ de timol em pó (Synth, SPLabor, Presidente Prudente, Brasil).

Após as fraturas, os espécimes foram observados em microscópico ótico Metrimpex (Metrimpex, Budapeste, Hungria) para identificação dos padrões de fraturas, os quais foram classificados em falha coesiva em substrato dental (esmalte ou dentina), coesiva em resina, adesiva ou mista ${ }^{23}$. Em seguida, as amostras foram observadas em microscópio confocal de escaneamento a laser (Lext OLS4000, Olympus, Japão) para se adquirir imagens dos tipos de fraturas.

As lentes de aumento do microscópio ótico eram utilizadas conforme a necessidade para a classificação das fraturas.

A média dos dados de resistência de união dos palitos provindos de um mesmo dente fizeram o valor médio daquele dente. A análise estatística foi realizada com 8 espécimes por 
grupo (unidade experimental foi 0 dente). Os dados foram analisados por ANOVA e "Tukey" $(\alpha=0.05)$ com o software SPSS 24 (IBM, Chicago, EUA). 

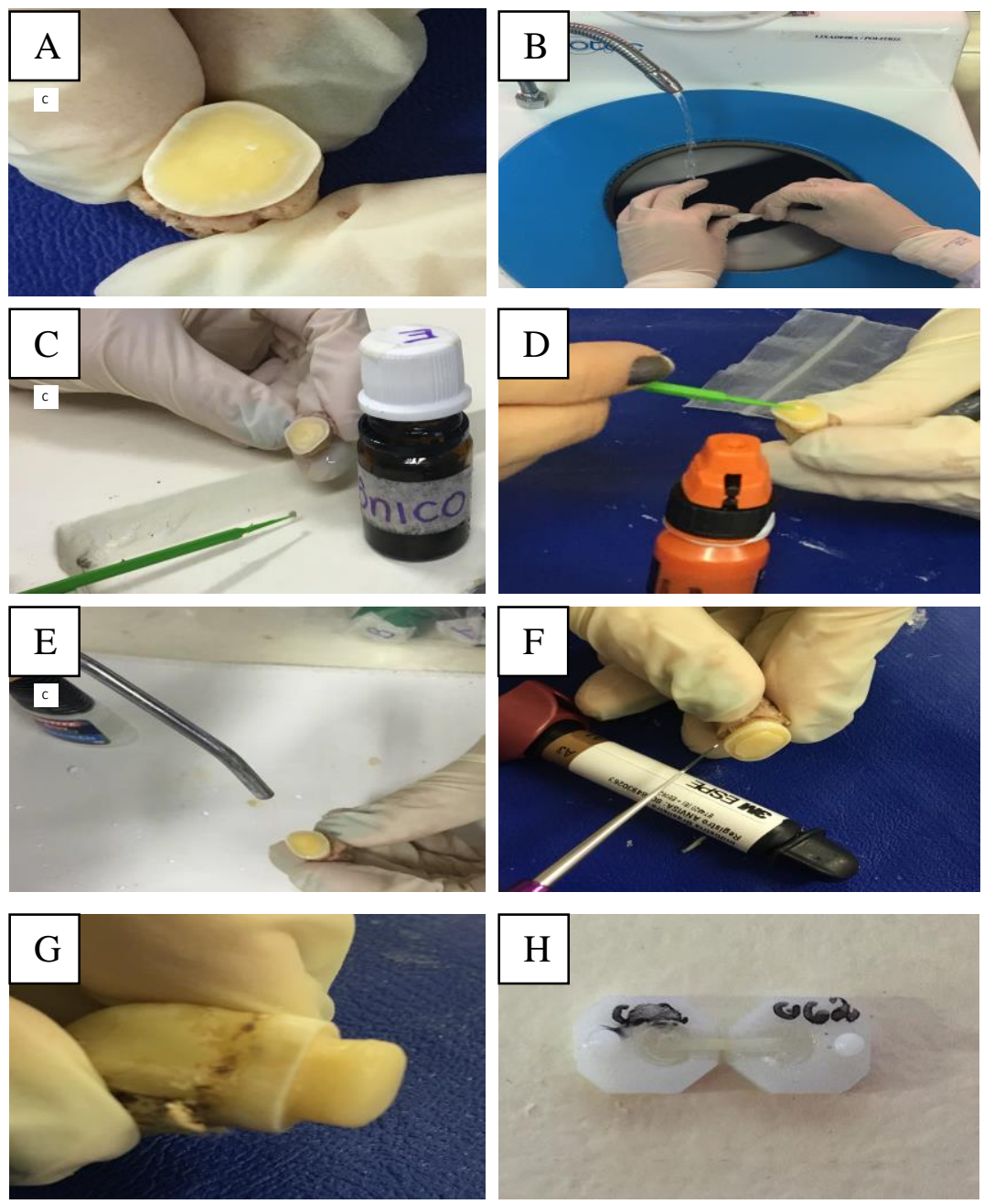

Figura 1. Passo a passo do preparo dos dentes humanos para o teste de microtração. A)Exposição dentinária. B)Simulação da lama dentinária. C)Condicionamento ácido de forma passiva. D)Aplicação do adesivo de forma ativa. E)Jato de ar para volatilização do solvente. F)Restauração. G) Restauração com $4 \mathrm{~mm}$ de altura. H)Palito no dispositivo Jig Geraldeli - Odeme. 


\section{Preparo dos Dentes Bovinos para Microcisalhamento}

Os dentes bovinos tiveram sua raiz removida na altura da junção amelocementária através de um disco diamantado (Erios, São Paulo, Brasil) sob refrigeração à água em uma máquina de corte (Micromet, Rockvill, Estados Unidos da América - EUA).

Cerca de $3 \mathrm{~mm}$ de altura da face incisal dos dentes bovinos foi removida com disco de carborundum (American Burrs, Palhoça, Santa Catarina, Brasil) instalada em peça reta e em micromotor (Kavo Dental, Joinville, Brasil).

Em seguida, cada um teve sua superfície vestibular polida, a fim de que fosse criada uma superfície de esmalte planificada. Isso se deu através de lixas d'água de granulação 400 (3M ESPE, Minessota, Estados Unidos da América) por 60 segundos sob a máquina Politriz Metalográfica (Teclago, São Paulo, Brasil).

Após as planificações, as superfícies foram desgastadas na mesma máquina Politriz com lixas d'água número 600 (3M ESPE, Minessota, Estados Unidos da América) por 60 segundos.

Cada superfície planificada foi embutida em canos de PVC - policloreto de vinila (Tigre, Rio Claro, Brasil) com resina acrílica autopolimerizável Jet (Clássico, São Paulo, Brasil).

As superfícies sofreram condicionamento ácido com os seguintes hidróxiácidos: ácido glicólico, tartárico, glucônico e gluconolactona de concentração de $37 \%$ por 30 segundos em esmalte.

As superfícies foram lavadas com água corrente por 10 segundos e completamente secas com jatos de ar por 15 segundos.

A aplicação de adesivo Adper Single Bond (3M ESPE, Minessota, Estados Unidos da América) foi feita de maneira similar aos dentes humanos. Em seguida, foi feita a fotopolimerização com Bluephase (Ivoclar Vivadent, Barueri, 
Brasil) do adesivo por 20 segundos com irradiância de 1.200 $\mathrm{mW} / \mathrm{cm}^{2}$.

Em seguida, 144 pedaços de macarrões Adria Furado número 5 foram medidos através de um paquímetro digital de fibra de carbono e cortados em uma altura de $1,5 \mathrm{~mm}$. Cada dente recebeu em média 4 macarrões que foram posicionados e preensados sobre as superficies dentárias de esmalte.

Alguns dentes receberam menos que 4 macarrões devido a irregularidade da superfície.

Depois de posicionados, os macarrões foram preenchidos com resina composta microhíbrida (Filtek Z250, 3M ESPE, Minessota, Estados Unidos da América) em um incremento único e fotopolimerizado por 40 segundos.

Em seguida, cada amostra foi colocada em um recipiente com água destilada (Reymer, Aparecida de Goiânia, Brasil) por 24 horas em uma estufa (Biodont, São Paulo, Brasil) de $27^{\circ} \mathrm{C}$, para que esses pudessem ser retirados.

Após 24 horas, as amostras foram retiradas da estufa e cada macarrão foi removido através de um estilete.

Em seguida, cada amostra foi levada à máquina de testes universal Shimadzu (Shimadzu Corp, Quioto, Japão), a fim de que fosse possível realizar os testes de resistência de união ao microcisalhamento entre as resinas e as superfícies. A velocidade utilizada na máquina foi de $0,5 \mathrm{~mm} / \mathrm{min}$.

A média dos dados de resistência de união ao microcisalhamento dos 4 cilindros provindos de um mesmo dente perfizeram o valor médio daquele dente. A análise estatística foi realizada com $n=8$ por grupo (unidade experimental foi o dente). Os dados foram analisados por Análise de Variância de um fator - ANOVA e Tukey $(\alpha=0,05)$ com o software SPSS 24 (IBM, Chicago, EUA).

Exatamente como nos molares humanos, após a fratura das amostras, os espécimes foram observados em microscópico ótico Metrimpex (Metrimpex, Budapeste, Hungria) para 
identificação dos padrões de fraturas, os quais foram classificados em falha coesiva em substrato dental (esmalte ou dentina), coesiva em resina, adesiva ou mista ${ }^{23}$.

Após a classificação, através de microscópio confocal de escaneamento a laser (Lext OLS4000, Olympus, Japão) obtevese imagens dos tipos de fraturas obtidas durante o teste. 



Figura 2: Passo a passo do preparo dos dentes bovinos para o teste de microcisalhamento. 1) Planificação dos dentes. 2) Lavagem após condicionamento ácido. 3) Macarrões após os cortes de $1,5 \mathrm{~mm}$. 4)Posicionamento dos macarrões em esmalte. 5) Incremento de resina sendo colocado. 6) Macarrões após terem sido retirados da estufa. 7) Remoção dos macarrões. 8) Teste de Microcisalhamento. 


\section{Resultados}

Os resultados do teste de resistência de união ao microcisalhamento (esmalte) são apresentados no Quadro 1.

Quadro 1: Resistência de União (MPa, DP) em esmalte em função do ácido utilizado.

\begin{tabular}{|c|c|}
\hline Ácidos & Resistência de União (MPa) $^{\text {Fosfórico (FOS) }}$ \\
\hline Glicólico (GLI) & $27,72(4,98)^{\mathrm{A}}$ \\
\hline Tartárico (TAR) & $23,90(4,15)^{\mathrm{A}}$ \\
\hline Gluconolactona (GLA) & $16,69(2,92)^{\mathrm{AB}}$ \\
\hline Glucônico (GLU) & $16,70(4,70)^{\mathrm{B}}$ \\
\hline
\end{tabular}

Letras diferentes entre grupos denotam diferença estatisticamente significativa.

Em relação aos valores de $\mathrm{RU}$ em esmalte obtidos pelos hidroxiácidos testados, percebeu-se que uma média superior sem diferença significativa foi observada entre os grupos FOS, GLI e TAR.

Um segundo grupamento estatístico reuniu os grupos TAR, GLU e GLA. Assim, os grupos FOS e GLI apresentaram resultados superiores a GLU e GLA. O grupo TAR não foi estatisticamente diferente de nenhum outro grupo.

Os resultados do teste de resistência de união a microtração (dentina) são apresentados no Quadro 2. 
Quadro 2: Resistência de União (MPa, DP) em dentina em função do ácido utilizado. Letras diferentes entre grupos denotam diferença estatisticamente significativa

\begin{tabular}{|c|c|}
\hline Ácidos & Resistência de União (MPa) $^{\text {Fosfórico }}$ \\
\hline Glicólico & $18,68(6,90)^{\mathrm{A}}$ \\
\hline Tartárico & $20,55(6,69)^{\mathrm{A}}$ \\
\hline Gluconolactona & $16,53(3,96)^{\mathrm{AB}}$ \\
\hline Glucônico & $10,17(2,74)^{\mathrm{B}}$ \\
\hline
\end{tabular}

Os ácidos GLI, FOS, TAR e GLU apresentaram maiores valores de RU à dentina. Os grupos TAR, GLU e GLA formaram um segundo grupamento estatístico. Assim, é possível afirmar que a RU à dentina de FOS e GLI foi superior a GLA.

Os espécimes fraturados foram observados em um microscópio ótico Metrimpex (Metrimpex, Budapeste, Hungria) para classificação das mesmas em falha coesiva em substrato dental (SD), coesiva em resina $(R)$, adesiva $(A)$ ou mista $(M)^{23}$. Os dados obtidos baseados nas classificações da resistência de união (RU) foram analisados em quadro. (Quadro 3)

Quadro 3. Quantidade de cada padrão de fratura em esmalte de cada ácido utilizado

\begin{tabular}{|c|c|c|c|c|c|}
\hline \multicolumn{1}{|c|}{ Ácidos } & SD (n) & $\mathbf{R}(\mathbf{n})$ & $\mathbf{A}(\mathbf{n})$ & $\mathbf{M}(\mathbf{n})$ & $\mathbf{( n )}$ \\
\hline & 0 & 0 & 30 & 0 & 30 \\
\hline FOS & 0 & 0 & 26 & 0 & 26 \\
\hline GLI & 0 & 0 & 24 & 0 & 24 \\
\hline TAR & 0 & 1 & 31 & 0 & 32 \\
\hline GLU & 0 & 3 & 29 & 0 & 32 \\
\hline GLA & 0 & TOTAL & 144 \\
\hline \multicolumn{7}{|c|}{}
\end{tabular}

Em relação à porcentagem de classificação de fraturas em esmalte, percebe-se que independente do condicionamento 
com o hidroxiácido utilizado, em geral, o maior padrão foi de caráter adesivo (A). (Quadro 4)

Quadro 4. Porcentagem da incidência de fratura em esmalte (\%).

\begin{tabular}{|c|c|c|c|c|}
\hline Ácidos & \multicolumn{5}{|c|}{} \\
\cline { 1 - 4 } & SD (\%) & $\mathbf{R}(\%)$ & $\mathbf{A}(\%)$ & $\mathbf{M}(\%)$ \\
\hline FOS & $0 \%$ & $0 \%$ & $100 \%$ & $0 \%$ \\
\hline GLI & $0 \%$ & $0 \%$ & $100 \%$ & $0 \%$ \\
\hline TAR & $0 \%$ & $0 \%$ & $100 \%$ & $0 \%$ \\
\hline GLA & $0 \%$ & $6,45 \%$ & $93,54 \%$ & $0 \%$ \\
\hline GLU & $0 \%$ & $3,13 \%$ & $96,87 \%$ & $0 \%$ \\
\hline \multicolumn{2}{|c|}{ TOTAL } & $\mathbf{2 , 1 \%}$ & $\mathbf{9 7 , 9 \%}$ & \\
& &
\end{tabular}

Enquanto os padrões de fraturas em esmalte foram apenas de algumas poucas falhas coesivas em resina $(R)$ e de uma grande maioria de falhas adesiva (A), aqueles que ocorreram em dentina englobaram outros tipos de classificação de fratura. (Quadro 5)

Quadro 5. Quantidade de cada padrão de fratura em dentina.

\begin{tabular}{|c|c|c|c|c|c|}
\hline Ácidos & SD (n) & $\mathbf{R}(\mathbf{n})$ & $\mathbf{A}(\mathbf{n})$ & $\mathbf{M}(\mathbf{n})$ & Total (n) \\
\hline & 0 & 1 & 36 & 1 & 38 \\
\hline FOS & 0 & 0 & 34 & 0 & 34 \\
\hline GLI & 1 & 2 & 31 & 2 & 36 \\
\hline TAR & 0 & 2 & 33 & 3 & 38 \\
\hline GLU & 1 & 0 & 35 & 1 & 37 \\
\hline GLA & \multicolumn{7}{|c|}{ TOTAL } \\
\hline \multicolumn{7}{|c|}{}
\end{tabular}

Através da incidência de fraturas em porcentagem, percebe-se, novamente, que embora existam outros tipos de 
fraturas em dentina a falha adesiva $(A)$ permanece sendo a mais comum. (Quadro 6)

Quadro 6. Porcentagem da incidência de fratura em dentina (\%).

\begin{tabular}{|c|c|c|c|c|}
\hline \multirow[t]{2}{*}{ Ácidos } & & & & \\
\hline & SD (\%) & R (\%) & $\mathbf{A}(\%)$ & M (\%) \\
\hline FOS & $0 \%$ & $2,63 \%$ & $94,73 \%$ & $2,63 \%$ \\
\hline GLI & $0 \%$ & $0 \%$ & $100 \%$ & $0 \%$ \\
\hline TAR & $2,77 \%$ & $5,55 \%$ & $86,11 \%$ & $5,55 \%$ \\
\hline GLA & $2,70 \%$ & $0 \%$ & $94,5 \%$ & $2,70 \%$ \\
\hline GLU & $0 \%$ & $5,26 \%$ & $86,8 \%$ & $7,89 \%$ \\
\hline TOTAL & $1,09 \%$ & $2,73 \%$ & $92,34 \%$ & $3,82 \%$ \\
\hline
\end{tabular}

A figura 3 apresenta imagens representativas de fraturas ocorridas nos testes de microtração (dentina) e microcisalhamento (esmalte). 


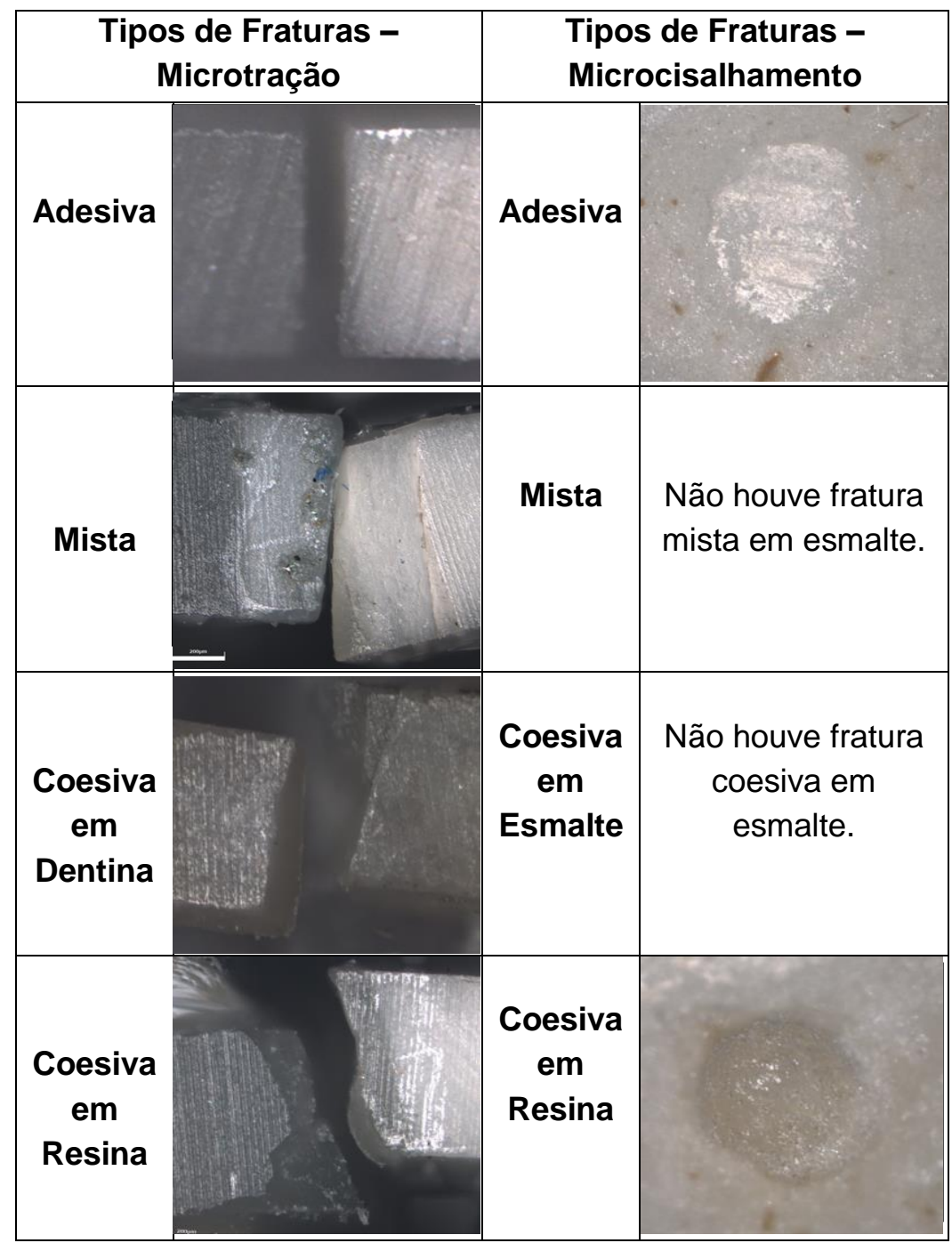

Figura 3: Quadro representativo de imagens da classificação dos tipos de fraturas obtidos através dos testes de microcisalhamento e microtração. 


\section{DISCUSSÃO}

Quando são analisados estudos de adesão com ácido fosfórico, vê-se que o condicionamento com esse ácido em esmalte apresenta resultados excelentes, como visto no estudo de Hashimoto et al., 2000. Entretanto, embora o ácido fosfórico em dentina relate histórico de adesão ${ }^{2}$, esse mostra alguns problemas, uma vez que ele tem a capacidade de desmineralizar de forma excessiva e profunda a dentina, e consequentemente, expõe as fibrilas de colágeno permitindo a inicialização do processo de degradação dessa substância, além da infiltração adesiva deficiente. ${ }^{25,34,35}$

Alguns estudos já sugeriram a possibilidade de alguns hidroxiácidos desmineralizarem de forma mais superficial $o$ esmalte e a dentina ${ }^{31}$, porém poucas referências que investiguem a influência desses ácidos nos substratos dentários são conhecidas. $^{28,31}$

Os resultados de adesão em esmalte obtidos no estudo propõem que hidroxiácidos, como o tartárico e o glicólico apresentam resultados similares ao ácido fosfórico (controle). Porém, ácidos como o glucônico e o gluconalacoctona possuem resultados inferiores ao grupo controle e ao ácido glicólico.

Em dentina, os ácidos fosfórico, glicólico, glucônico e tartárico fazem parte de um mesmo grupo estatístico. Entretanto, apenas os ácidos glicólico e fosfórico foram superiores a gluconolactona.

Ao serem analisados os padrões de fraturas dos testes, os dados relatam que a classificação mais frequente foi a de falhas adesivas, com uma incidência equivalente à $92,34 \%$ em dentina e $97,9 \%$ em esmalte. Isso mostra que as forças dos testes de microtração e microcisalhamento utilizados nas amostras realmente verificou a resistência de união entre a interface adesiva e o referido substrato, cumprindo o propósito do trabalho. ${ }^{27}$ 
Ao avaliar os resultados obtidos em esmalte e em dentina, percebe-se que em ambos o ácido glicólico acompanha o padrão de resultados obtidos pelo ácido fosfórico, que é o grupo controle.

Outros estudos do nosso grupo de pesquisa da Universidade de Brasília - UnB (ainda não publicados) indicam que o ácido glicólico apontou uma capacidade de aumentar a rugosidade de esmalte e de dentina, porém com uma menor profundidade de desmineralização em dentina e em esmalte, quando comparados ao ácido fosfórico.

Sabendo disso e associado aos resultados promissores apresentados pelo ácido glicólico em esmalte e em dentina deste trabalho, sugere-se uma realização de mais estudos para avaliar o potencial uso de ácido glicólico na adesão em esmalte e em dentina através da técnica de condicionamento ácido total. 


\section{CONSIDERAÇÕES FINAIS}

O condicionamento ácido é a primeira etapa do passo a passo restaurador na técnica etch and rinse e tem como objetivo garantir a desmineralização dos substratos dentais para a adequada infiltração dos sistemas adesivos, para que se obtenha uma região com microporosidades e ofereça uma retenção às restaurações.

Após o trabalho, foi possível concluir que alguns dos hidroxiácidos testados apresentaram resultados de resistência de união ao esmalte e à dentina similares; embora o ácido fosfórico seja utilizado como atual padrão de condicionamento na Odontologia.

Os diferentes hidroxiácidos testados podem promover a adesão do esmalte e da dentina, quando é seguido o protocolo adesivo convencional.

Alguns hidroxiácidos apresentaram valores similares e outros apresentaram valores mais altos quando comparados ao ácido fosfórico. Assim, o condicionamento de esmalte e de dentina pode ser realizado por hidroxiácidos alternativos ao ácido fosfórico.

Entre os hidroxiácidos testados, a resistência de união em dentina e em esmalte promovida pelo ácido glicólico e pelo ácido tartárico foi semelhante a do ácido fosfórico. Destaca-se o potencial do ácido glicólico, pela consistência de resultados superiores a outros hidroxiácidos testados. 


\section{REFERÊNCIAS}

1. Buonocore MG. A Simple Method of Increasing the Adhesion of Acrylic Filling Materials to Enamel Surfaces. J Dent Res. 1955 Dec; 34:849-53.

2. Nakabayashi N, Kojima K, Masuhara E. The promotion of adhesion by the infiltration of monomers into tooth substrates. $J$ Biomed Mater Res. 1982 May;16:265-73.

3. Hashimoto M, Ohno H, Kaga M, Endo K, Sano H, Oguchi $\mathrm{H}$. In vivo degradation of resin-dentin bonds in humans over 1 to 3 years. J Dent Res. 2000 Jun;79:1385-91.

4. Carrilho MRO, Geraldeli S, Tay F, de Goes MF, Carvalho RM, Tjäderhane L, et al. In vivo preservation of the hybrid layer by chlorhexidine. J Dent Res. 2007 Jun;86:529-33.

5. Carrilho MRO, Carvalho RM, de Goes MF, di Hipólito V, Geraldeli S, Tay FR, et al. Chlorhexidine preserves dentin bond in vitro. J Dent Res. 2007 Jan;86:90-4.

6. Tjäderhane L, Nascimento FD, Breschi L, Mazzoni A, Tersariol ILS, Geraldeli S, et al. trategies to prevent hydrolytic degradation of the hybrid layer-A review. Dent Mater. 2013 Oct;29:999-1011.

7. Anchieta RB, Machado LS, Martini AP, Santos PH dos, Giannini M, Janal M, Tovar N, Sundfeld RH, Rocha EP, Coelho PG. Effect of long-term storage on nanomechanical and morphological properties of dentin-adhesive interfaces. Dent Mater. 2015 Feb;31:141-53.

8. Van Meerbeek B, De Munck J, Yoshida Y, Inoue S, Vargas M, Vijay P, Van Landuyt K, Lambrechts P, Vanherle G. Buonocore memorial lecture. Adhesion to enamel and dentin: current status and future challenges. Oper Dent. 2003 Jun;28:215-35. 
9. Van Meerbeek M, Ohno H, Sano H, Kaga M, Oguchi H. In vitro degradation of resin- dentin bonds analyzed by microtensile bond test, scanning and transmission eléctron microscopy. Biomaterials. 2003 Sep;24:3795-803.

10. Takahashi M1, Nakajima M, Tagami J, Scheffel DL, Carvalho RM, Mazzoni A, Cadenaro M, Tezvergil-Mutluay A, Breschi L, Tjäderhane L, Jang SS, Tay FR, Agee KA, Pashley $\mathrm{DH}$. The importance of size-exclusion characteristics of type I collagen in bonding to dentin matrices. Acta Biomater. 2013 Dec;9:9522-8.

11. Mazzoni A, Pashley DH, Ruggeri A, Vita F, Falconi M, Di Lenarda R, Breschi L. Adhesion to chondroitinase ABC treated dentin. J Biomed Mater Res B Appl Biomater. 2008 Jul;86:22836.

12. Bedran-Russo AKB, Pereira PNR, Duarte WR, Okuyama $\mathrm{K}$, Yamauchi M. Removal of dentin matrix proteoglycans by trypsin digestion and its effect on dentin bonding. J Biomed Mater Res B Appl Biomater. 2008 Apr;85:261-6.

13. Bertassoni LE, Orgel JPR, Antipova O, Swain MV. The dentin organic matrix - limitations of restorative dentistry hidden on the nanometer scale. Acta iomater. 2012 Jul;8:2419-33.

14. Balooch M, Habelitz S, Kinney JH, Marshall SJ, Marshall GW. Mechanical properties of mineralized collagen fibrils as influenced by demineralization. J Struct Biol. 2008 Jun;162:40410.

15. Van Scott EJ, Ditre CM, Yu RJ. Alpha-hydroxyacids in the treatment of signs of photoaging. Clin Dermatol. 1996 Apr;14:217-26.

16. Inan $\mathrm{S}$, Oztukcan $\mathrm{S}$, Vatansever $\mathrm{S}$, Ermertcan AT, Zeybek D, Oksal A, Giray G, Muftuoglu S. Histopathological and ultrastructural effects of glycolic acid on rat skin. Acta Histochem. 2006;108:37-47.24. 
17. Campos EA, Saad JRC, Oliveira Júnior OB, Porto Neto ST, Campos LA, Andrade MF. Effect of delayed insertion of composite resin the bond strength of self-etching adhesive systems. 2009; RSBO v. 6, n. 3.

18. Rito C, Maceira JP. Reflectance confocal microscopy in the diagnosis of cutaneous melanoma. An Bras Dermatol. 2009;84:636-42.

19. Oliveira NA, Diniz LSM, Svizero NR, Alpino PHP, Pegoraro CACCP. Dental Adhesives: new concepts and clinical applications. Revista Dentística on line - ano 9, número 19, 2010.

20. Guarda MB. Tratamentos da Superfície da resina composta indireta: Efeito na resistência da união e análise por microscopia confocal de varredura a laser. Piracicaba, SP: [s.n.], 2013.

21. Toledano M., Osorio R, Perdigao J, Rosales JI, Thompson JY, Cabrerizo-Vilchez MA. Effect of acid ething and collagen removal on dentin wettability and roughness. J.Biomed Mater Res., 1999 Nov;47:198-203.

22. Matos AB, Palma RG, Saraceni $\mathrm{CH}$, Matson E. Effects of acid etching on dentin surface: SEM morphological study. Braz Dent J. 1997;8:35-41.

23. Pashley DH, Ciucchi B, Sano H, Horner JA. Permeability of dentin to adhesive agents. Quintessence Int. 1993 Sep; 24:618-31.

24. Castilio, D. Influência dos espessantes na rugosidade da superfície da dentina e resistência ao cisalhamento de três sistemas adesivos. Tese de Doutorado - Faculdade de Odontologia da Universidade de São Paulo. Bauru, 2005. 159p:il.;30 cm.

25. Retief DH, Mandras RS, Russell CM, Denys FR. Phosphoric acid as dentin etchant. Am J Dent. 1992 Feb;5:24-8. 
26. Vermelho, PM. Sistemas adesivos universais: resistência de união ao esmalte e dentina, padrão de fratura e análise ultramorfológica. Tese de doutorado - Unicamp. Piracicaba, 2015.

27. Ribeiro, JCV. Ensaio de microtração na avaliação da resistência adesiva: fundamentos e aplicações. RGO - Rev Gaúcha Odontol., Porto Alegre, v.61, p. 497-504, jul./dez., 2013

28. Bringhenti IL, Leal LO, Bernardi JB, Farina AP, Cecchin D. Resistência de União de pinos de fibra à dentina radicular condicionada com ácido Glicólico. IV Semana do Conhecimento. Universidade de Passo Fundo. 2017.

29. Nardin P, Guterres S. Alfa-Hidroxiácidos: Aplicações Cosméticas e Dermatológicas. Caderno de Farmácia, v.15, n.1, p.7-14, 1999.

30. Youssef JA, Turbino ML, Youssef MN, Matson E. Resistência de união à dentina de resinas compostas associadas a sistemas adesivos com e sem carga. Pesqui Odontol Bras v. 15 , n. 2, p. 157-160, abr./jun. 2001

31. Ramachandran S, Fontanille P, Pandey A, Larroche C. Gluconic Acid: Properties, Applications and Microbial Production. Biotechnol. 2006; 44 185-195.

32. Breschi L, Mazzoni A, Ruggeri A, Cadenaro M, Di Lenarda R, Dorigo ES. Dental adhesion review: aging and stability of the bonded interface. Dent. Mat., v.24, n.1, p.90-101, abr.2008.

33. Kinney JH, Marshall SJ, Marshall GW. The mechanical properties of human dentin: a critical review and re-evaluation of the dental literature. Crit Rev Oral Biol Med. 2003;14:13-29.

34. Linde A, Goldberg M. Dentinogenesis. Crit Rev Oral Biol Med; 1993. 4:679-728. 
35. Reis AF, Pereira PNR, Giannini M. Sistemas Adesivos Atualidades e Perspectivas. Ebook. Jubileu de Ouro. 25 CIOSP. 2007. 


\section{NORMAS DA REVISTA}

A Journal of Dentistry é o jornal odontológico internacional líder no campo da restauração odontológica. Colocando ênfase na publicação de trabalhos de pesquisa novos e de alta qualidade, o Journal tem como objetivo influenciar a prática da odontologia em nível clínico, de pesquisa, de indústria e em um nível base internacional.

Os tópicos abordados incluem o gerenciamento de doenças dentárias, periodontologia, endodontia, odontologia operatória, prótese dentária fixa e removível, ciência de biomateriais dentários, ensaios clínicos a longo prazo, incluindo epidemiologia e saúde bucal, transferência de tecnologia de novos instrumentos ou procedimentos, bem como biologia oral clinicamente relevante e pesquisa translacional.

O Journal of Dentistry publicará trabalhos de pesquisa científica originais, incluindo comunicações curtas. Também está interessada em publicar artigos e líderes em áreas temáticas que serão ligadas a nova pesquisa científica. Os anais da conferência são bem-vindos e as manifestações de interesse são comunicados ao editor.

- Público:

Os interessados em desenvolvimentos em pesquisa oral e odontológica, incluindo clínicos, dentistas investigadores, acadêmicos clínicos, envolvidos na indústria dentária e pessoas relevantes para a prática de odontologia.

- Fator de Impacto: 2016: 3.456

- Submissões:

Solicita-se aos autores que enviem seus manuscritos e números originais por meio da submissão on-line. Usando este sistema on-line, os autores podem submeter manuscritos e 
acompanhar o seu progresso através do sistema para publicação.

\section{- Tipos de papel}

As contribuições que se enquadrem nas seguintes categorias serão consideradas para publicação:

- Relatório de Pesquisa Original: tamanho máximo 6 páginas impressas aproximadamente 20 páginas datilografadas, incluindo ilustrações e tabelas.

- Artigos de revisão: tamanho máximo de 10 páginas impressas, aproximadamente 33 páginas datilografadas, incluindo ilustrações e tabelas.

- Comunicação breve para publicação rápida: comprimento máximo 2 páginas impressas, aproximadamente 7 páginas datilografadas, incluindo ilustrações.

- Cartas com comentários informados e críticas construtivas ao material previamente publicado em o jornal.

Todos os textos digitalizados devem ser acompanhados por uma Nota de permissão. Esta é uma carta assinada por cada autor (não apenas o autor correspondente), afirmando que o artigo foi submetido exclusivamente ao Journal of Dentistry e que não está concorrentemente sob consideração para publicação em outro periódico.

Os autores em perspectiva devem confirmar que o trabalho submetido, incluindo imagens, é original. Autores devem ser lembrados que se as imagens incluídas (por exemplo, Tabelas e Figuras) tiverem sido publicadas anteriormente, exigem permissão de direitos autorais.

Observe que o Journal of Dentistry não aceita relatos de casos e estes serão removidos do sistema, se enviado. 
Somente as pessoas que contribuíram significativamente para o manuscrito submetido devem ser listado como autores. $O$ Editor-Chefe espera que um manuscrito normalmente não tenha mais mais de 6 autores, a menos que um caso seja feito pelo autor correspondente dentro da carta de apresentação incluir outros autores. Todos os autores nomeados deveriam estar envolvidos no trabalho que leva a publicação do artigo e deveria ter lido $o$ artigo antes de ser submetido para publicação.

- Lista de verificação de envio

Você pode usar essa lista para realizar uma verificação final do seu envio antes de enviá-lo para o periódico. Reveja. Por favor, verifique a seção relevante neste Guia para Autores para mais detalhes.

Assegure-se de que os seguintes itens estejam presentes:

Um autor foi designado como o autor correspondente com detalhes de contato:

- Endereço de e-mail

- Endereço postal completo

Todos os arquivos necessários foram enviados:

- Manuscrito:

- incluir palavras-chave

- Todas as figuras (incluem legendas relevantes)

- Todas as tabelas (incluindo títulos, descrição, notas de rodapé)

- Garantir que todas as citações de figura e tabela no texto correspondam aos arquivos fornecidos

- Indique claramente se a cor deve ser usada para quaisquer figuras impressas Arquivos Gráficos Resumos / 
Destaques (quando aplicável); Arquivos suplementares (quando aplicável).

- Outras considerações:

- O manuscrito foi 'verificado ortograficamente' e 'verificado gramática';

- Todas as referências mencionadas na Lista de Referência são citadas no texto e vice-versa;

- Foi obtida permissão para o uso de material protegido por direitos autorais de outras fontes (incluindo Internet);

- Uma declaração de interesses conflitantes é fornecida, mesmo que os autores não tenham interesses declarar;

- As políticas de diário detalhadas neste guia foram revisadas;

- Sugestões de árbitros e detalhes de contato fornecidos, com base nos requisitos da revista.

- Informação essencial da página de rosto:

- Título. Conciso e informativo. Títulos são frequentemente usados em sistemas de recuperação de informações. Evitar abreviaturas e fórmulas, sempre que possível.

- Indicar claramente os nomes e sobrenomes de cada autor e verifique se todos os nomes estão escritos com precisão. Apresentar onde 0 trabalho real foi feito abaixo dos nomes. Indique todas as afiliações com letras imediatamente após o nome do autor e em frente ao endereço apropriado.

Forneça o endereço postal completo de cada pessoa, incluindo o nome do país e, se disponível, o Endereço de e-mail de cada autor. 
- Autor correspondente. Indique claramente quem irá lidar com a correspondência em todas as etapas da arbitragem e publicação, também pós-publicação. Esta responsabilidade inclui responder a quaisquer futuras consultas sobre Metodologia e Materiais. Assegure-se de que o endereço de e-mail seja fornecido e que os detalhes de contato são mantidos atualizados pelo autor correspondente.

- Endereço presente / permanente. Se um autor se mudou desde que o trabalho descrito no artigo foi feito, ou estava visitando no momento, um 'endereço atual' (ou 'endereço permanente') pode ser indicado como uma nota de rodapé ao nome desse autor. $O$ endereço no qual o autor realmente fez o trabalho deve ser retido como o principal endereço de afiliação. Números árabes sobrescritos são usados para tais notas de rodapé.

- A página de título deve conter as seguintes informações:

- Título do papel

- Título curto

- Nome (s), cargo e endereço (s) do (s) autor (es) (não são necessários graus académicos)

- Nome, endereço, telefone, fax e e-mail do autor correspondente

- até 6 palavras-chave

Ortografia: Inglês internacional.

Os autores são convidados a escrever o mais conciso possível.

O estilo do Journal of Dentistry requer que os artigos sejam organizados nas seguintes ordem: Título, Resumo, Introdução, Materiais e Métodos, Resultados, Discussão, Conclusões, Agradecimentos, Referências, Tabelas, Figuras.

Uma carta de apresentação deve acompanhar o novo submissão do manuscrito, dentro do qual os autores devem indicar a significância do trabalho apresentou em um comunicado não mais de 100 palavras. Uma nota de permissão assinada (detalhes abaixo) deve também ser incluído. 
Resumo: não deve exceder 250 palavras e deve ser apresentado nas seguintes subposições: Objetivos, Métodos; Resultados; Conclusões (Para Revisões: Objetivos; Dados; Fontes; Seleção de estudos;Conclusões).

Uma declaração de 50 palavras 'Clinical Significance' deve aparecer no final do resumo aconselhar os leitores sobre a importância clínica e relevância do seu trabalho. Estas subposições devem aparecem no texto do resumo.

Repetir o título do artigo no topo da página abstrata.

- Introdução: deve ser apresentada em formato estruturado, abrangendo os seguintes assuntos, não nas subposições: afirmações sucintas da questão em questão e a essência das conhecimento e compreensão pertinentes à questão. De acordo com o estilo de casa do Journal of Dentistry, o parágrafo final da introdução deve indicar claramente os objetivos e / ou objetivos datrabalho sendo relatado.

- Palavras-chave: até 6 palavras-chave devem ser fornecidas, usando ortografia britânica e evitando termos gerais e plurais e vários conceitos (evite, por exemplo, 'e', 'de') e sem abreviaturas.

- Abreviaturas e siglas: termos e nomes a serem referidos na forma de abreviaturas ou siglas devem ser dadas na íntegra quando mencionado pela primeira vez.

- Unidades: unidades SI devem ser usadas por toda parte. Se unidades não SI devem ser citadas, o equivalente SI deve siga imediatamente entre parênteses.

- Os nomes completos dos dentes individuais devem ser dados no teste. Em tabelas e lendas para ilustrações dentes 
individuais devem ser identificados usando o sistema de dois dígitos do IDE.

Estatisticas: os métodos estatísticos devem ser descritos com detalhes suficientes para permitir que um leitor experiente acesso aos dados originais para verificar os resultados reportados. Quando possível, os achados devem ser quantificados e medidas apropriadas de erro ou incerteza (como intervalos de confiança) dadas. Detalhes sobre critérios de elegibilidade para os sujeitos, randomização e o número de observações devem ser incluídos. O software informático eo (s) método (s) estatístico (s) utilizado (s) devem ser especificados com referências a funciona quando possível (com páginas especificadas).

- Ilustrações: devem ser enviadas eletronicamente usando um software comercial apropriado. Ajustes de brilho, contraste ou balanço de cores são aceitáveis. Tamanho da imagem: forneça uma imagem com um mínimo de $531 \times 1328$ pixels $(h \times w)$ ou proporcionalmente mais. A imagem deve ser legível em um tamanho de $5 \times 13 \mathrm{~cm}$ usando uma resolução de tela regular de 96 dpi. Tipos de arquivos preferidos: arquivos TIFF, EPS, PDF ou MS Office.

- Palavras-chave: Fornecer no máximo 6 palavras-chave

- Formatação de fontes de financiamento: Quando o financiamento for proveniente de uma bolsa disponivel para uma universidade, envie o nome do instituto que forneceu 0 financiamento.

- Tabelas: tabelas como texto editável e não como imagens. Tabelas podem ser colocadas ao lado do texto relevante no artigo, ou em página (s) separada (s) no final. 
- Referências:

1- Citação no texto:

Certifique-se de que todas as referências citadas no texto também estejam presentes na lista de referências (e vice-versa). versa). Quaisquer referências citadas no resumo devem ser dadas na íntegra. Resultados não publicados e pessoais as comunicações não são recomendadas na lista de referências, mas podem ser mencionadas no texto. Se estas referências estão incluídas na lista de referências devem seguir o estilo de referência padrão do revista e deve incluir uma substituição da data de publicação com 'Resultados não publicados' ou 'Comunicação pessoal'. A citação de uma referência como 'in press' implica que o item foi aceito para publicação.

\section{2-Links de referência:}

A maior capacidade de descoberta da pesquisa e a revisão por pares de alta qualidade são asseguradas por links on-line para as fontes citadas. Para nos permitir criar links para serviços de abstração e indexação, como Scopus, CrossRef e PubMed, certifique-se de que os dados fornecidos nas referências estejam corretos. Por favor observe que sobrenomes incorretos, títulos de periódicos / livros, ano de publicação e paginação podem impedir criação. Ao copiar referências, tenha cuidado, pois elas já podem conter erros.

Um DOI pode ser usado para citar e vincular artigos eletrônicos onde um artigo é publicado e citação completa. detalhes ainda não são conhecidos, mas o artigo está disponível online. Um DOI é garantido para nunca mudar, então você pode usá-lo como um link permanente para qualquer artigo eletrônico.

\section{3-Referências da Web}

No mínimo, o URL completo deve ser fornecido e a data em que a referência foi acessada pela última vez. Quaisquer mais informações, se conhecidas (DOI, nomes de autores, datas, 
referência a uma publicação de origem, etc.), também deve ser dado. Referências na Web podem ser listadas separadamente (por exemplo, após a lista de referência) sob um cabeçalho diferente, se desejado, ou pode ser incluído na lista de referência.

4-Referências de dados

As referências de dados devem incluir os seguintes elementos: nome (s) do autor, título do conjunto de dados, repositório de dados, versão (quando disponível), ano, e identificador persistente global. 